\title{
THE CLASSIFICATION OF STUNTED PROJECTIVE SPACES BY STABLE HOMOTOPY TYPE
}

\author{
BY
}

\author{
S. FEDER AND S. GITLER
}

\begin{abstract}
A complete classification of stable homotopy types of complex and quaternionic stunted projective spaces, denoted by $\mathbf{C} P_{n}^{k}$ and $Q P_{n}^{k}$ respectively, is obtained. The necessary conditions for such equivalences are found using $K$-theory and various characteristic classes introduced originally by J. F. Adams. As a by-product one finds the $J$-orders of the Hopf bundles over $\mathbf{C} P^{n}$ and $Q P^{n}$ respectively. The algebraic part is rather involved. Finally a homotopy theoretical argument yields the constructions of such homotopy equivalences as are allowed by the fulfillment of the necessary conditions.
\end{abstract}

1. Introduction. The results of this paper were announced in [9].

Let $F P^{n}$ be the projective space of dimension $n$ over $F$, where $F$ is either the field of complex numbers $\mathbf{C}$ or the quaternions $\mathbf{Q}$. We have the natural cofibrations

$$
F P^{m-1} \rightarrow F P^{n} \rightarrow F P_{m}^{n}
$$

for $m \leqslant n$.

Let $\xi$ denote the Hopf bundle over $F P^{n}$. It is well known that the stunted projective space $F P_{k}^{n+k}$ can be identified with the Thom space of $k \xi$ over $F P^{n}$ which we denote by $\left(F P^{n}\right)^{k \xi}$.

In this paper we study the stable homotopy type of stunted projective spaces $F P_{k}^{n+k}$ for $F=\mathbf{C}$ and $F=\mathbf{Q}$ and obtain a complete classification. This classification is given in terms of the $J$-orders of the Hopf bundles. We denote by $A_{n}$ the $J$-order of the Hopf bundle over $\mathbf{C} P^{n}$ and by $B_{n}$ the $J$-order of the quaternion Hopf bundle over $\mathbf{Q} P^{n}$. With this notation the classification can be stated as follows:

THEOREM 1.1. The spaces $\mathbf{C} P_{k}^{n+k}$ and $\mathbf{C} P_{l}^{n+l}$ are of the same stable homotopy type if and only if one of the following conditions hold $(n \neq 2,4)$ :

(i) $k-l \equiv O\left(A_{n}\right)$,

(ii) $k-l \equiv O\left(A_{n-1}\right)$ and $k+l \equiv O\left(A_{n}\right)$,

Received by the editors October 2, 1974.

AMS (MOS) subject classifications (1970). Primary 55D15, 55F50, 55G25.

$K e y$ words and phrases. Projective space, stunted projective space, Thom space, $J$-order, $K$ theory, spherical fibrations. 
(iii) $k-l \equiv O\left(A_{n-1}\right)$ and $k+l+2(n+1) \equiv O\left(A_{n}\right)$.

THEOREM 1.2. The spaces $\mathbf{Q} P_{k}^{n+k}$ and $\mathbf{Q} P_{l}^{n+l}$ are of the same stable homotopy type if and only if one of the following conditions hold:

(i) $k-l \equiv O\left(B_{n}\right)$,

(ii) $k-l \equiv O\left(B_{n-1}\right)$ and $k+l \equiv O\left(B_{n}\right)$.

The apparent difference between the two statements is due to the fact that the family of stunted complex projective spaces is closed under $S$-duality while the family of stunted quaternionic projective spaces is not. Note that conditions (ii) and (iii) in Theorem 1.1 correspond under $S$-duality.

In [6] we have proven that the conditions in Theorem 1.1 are necessary and have completed the classification for $n$ odd by observing that $A_{n}=A_{n-1}$ for that case. This paper thus completes and extends [6]. We also give a proof of the result of F. Sigrist and U. Sutter [15] on the number $B_{n}$.

Let $\nu_{p}(m)$ be the largest integer for which $p^{\nu_{p}(m)}$ divides $m$. The result is

THEOREM 3.4. The J-order $B_{n}$ of the Hopf bundle over $\mathbf{Q} P^{n}$ is given by

$$
B_{n}=\prod p^{p_{p}\left(B_{n}\right)}
$$

where

$$
\nu_{2}\left(B_{n}\right)=\max \left\{2 n+1,2 j+\nu_{2}(j) \mid 1 \leqslant j \leqslant n\right\}
$$

and

$$
\nu_{p}\left(B_{n}\right)=\max \left\{j+\nu_{p}(j) \mid 1 \leqslant j \leqslant 2 n /(p-1)\right\}
$$

when $p$ is an odd prime.

In [6] we used the fact, proven by Adams and Walker [3], that $A_{n}=M_{n+1}$, the Atiyah-Todd number. In this paper we shall also give a short proof of that fact.

The classification is accomplished in two steps. First, similar to what was done in [6], certain necessary conditions are obtained. This is achieved by using certain characteristic classes and the following simple proposition:

Proposition 1.4. If $f: X \rightarrow Y$ is a homotopy equivalence then the diagram

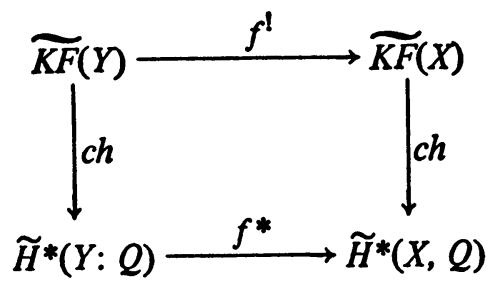

is commutative and both $f^{*}$ and $f^{!}$are invertible transformations. 
Here $\widetilde{K F}$ may stand for real $(F=O)$, complex $(F=U)$, or sympletic $(F=\mathrm{Sp})$ reduced $K$-theory and the Chern character is interpreted in the appropriate way.

Once the necessary conditions are obtained their sufficiency is established by constructing the desired homotopy equivalences. The low-dimensional cases present some anomalies and must be treated separately.

As corollaries we also obtain the representations

$$
\mathcal{E}\left(F P_{n}^{m}\right) \rightarrow \operatorname{Aut}\left(H^{*}\left(F P_{n}^{m}, Z\right)\right)
$$

where $\varepsilon(X)$ is the group of stable homotopy equivalences of $X$ and $F$ $=\mathbf{C}$ or $\mathbf{Q}$.

2. Characteristic classes. Let $\xi$ be a vector bundle over $X$. We say that $\xi$ is $K F$-orientable if there exists an isomorphism $K F(X) \stackrel{\phi}{\rightarrow} \widetilde{K F}\left(X^{\xi}\right)$. A choice of one such isomorphism is a $K F$-orientation. To simplify our exposition we shall say, unless otherwise stated, that $\xi$ is $K O$-orientable if $\xi$ has a $\operatorname{Spin}(8 n)$ structure and that $\xi$ is $K U$-orientable if $\xi$ has a $U(n)$-structure.

Let $U^{H}$ and $U^{F}$ denote the Thom classes for integral cohomology $H^{*}$ and $\widetilde{K F}$-theory respectively. Adams in [2] defines a class $b h(\xi) \in H^{*}(X ; Q)$ by

$$
\operatorname{ch} U^{F}=U^{H} \cup b h(\xi)
$$

where the character is taken after complexification if $K O$ is the theory in question. Adams also defines classes $\rho^{k}(\xi)$ (these are due to Bott [5]) by

$$
\psi^{k} U^{F}=U^{F} \rho^{k}(\xi) \text {. }
$$

If $\xi$ and $\eta$ are vector bundles which are $J$-equivalent then their classes $b h$ and $\rho^{k}$ satisfy

$$
\begin{aligned}
& b h(\xi)=b h(\eta) c h(1+x), \\
& \rho^{k}(\xi) \stackrel{k}{=} \rho^{k}(\eta) \psi^{k}(1+x) /(1+x) .
\end{aligned}
$$

for some element $x \in \widetilde{K F}(X)$, and all $k$. The equality (2.2) denoted by $=^{k}$ means that the two sides may differ by a factor $k^{r}$ for some $r$.

The solution of the Adams conjecture by Adams [1] and Quillen [13] establishes (2.2) as a necessary and sufficient condition for two real vector bundles to be $J$-equivalent.

In special cases conditions (2.1) and (2.2) are equivalent. Let $F=O$ or $U$.

Proposition 2.3. Suppose $K F(X)$ has no torsion. If $\xi_{1}$ and $\xi_{2}$ are $K F$ orientable vector bundles and if there exists a class $x \in K F(X)$ such that $b h\left(\xi_{1}\right)=b h\left(\xi_{2}\right) \operatorname{ch}(1+x)$, then for all integers $k \neq 1$, we have: $\rho^{k}\left(\xi_{1}\right)$ $={ }^{k} \rho^{k}\left(\xi_{2}\right) \psi^{k}(1+x) /(1+x)$. 
Proof. Recall the definition of $\bar{\psi}^{k}: H^{\mathrm{ev}}(X ; Q) \rightarrow H^{\mathrm{ev}}(X ; Q)$ by $\bar{\psi}^{k}(u)$ $=k^{1 / 2 \operatorname{dim} u} u$. Then because of the hypothesis, we have $\operatorname{ch} \psi^{k}=\bar{\psi}^{k} c h$.

Let $\xi$ be a $K F$-orientable bundle of real dimension $2 n$. Then

$$
\operatorname{ch} \psi^{k} U_{\xi}^{F}=\bar{\psi}^{k} \operatorname{ch} U_{\xi}^{F}=\bar{\psi}^{k}\left(U_{\xi}^{H} \cdot b h(\xi)\right)=k^{n} U_{\xi}^{H} \bar{\psi}^{k}(b h(\xi)) .
$$

Also

$$
\operatorname{ch} \psi^{k} U_{\xi}^{F}=\operatorname{ch}\left(U_{\xi}^{F} \cdot \rho^{k}(\xi)\right)=U_{\xi}^{H} \cdot b h(\xi) \operatorname{ch} \rho^{k}(\xi) .
$$

Hence

$$
k^{n} \bar{\psi}^{k}(b h(\xi))=b h(\xi) \operatorname{ch} \rho^{k}(\xi) .
$$

Now consider $K F$-orientable bundles $\xi_{1}$ and $\xi_{2}$ of dimensions $2 n_{1}$ and $2 n_{2}$ respectively. We have

$$
\operatorname{bh}\left(\xi_{1}\right) \operatorname{ch} \rho^{k}\left(\xi_{1}\right)=b h\left(\xi_{2}\right) \operatorname{ch}\left[(1+x) \rho^{k}\left(\xi_{1}\right)\right]
$$

and

$$
\begin{aligned}
k^{n_{1}} \bar{\psi}^{k}\left(b h\left(\xi_{1}\right)\right) & =k^{n_{1}} \bar{\psi}^{k}\left(b h\left(\xi_{2}\right) \operatorname{ch}(1+x)\right) \\
& =k^{n_{1}-n_{2}} \operatorname{bh}\left(\xi_{2}\right) \operatorname{ch} \rho^{k}\left(\xi_{2}\right) \bar{\psi}^{k} \operatorname{ch}(1+x) .
\end{aligned}
$$

By the above, (i) and (ii) are equal. Now concentrating on the right-hand sides of (i) and (ii), we have

$$
b h\left(\xi_{2}\right) \operatorname{ch}\left[(1+x) \rho^{k}\left(\xi_{1}\right)-k^{n_{1}-n_{2}} \rho^{k}\left(\xi_{2}\right) \psi^{k}(1+x)\right]=0 .
$$

But $b h\left(\xi_{2}\right) c h y=0$ if and only if $y=0$, because, $\operatorname{ch}\left(U_{\xi_{2}}^{F} \cdot y\right)=U_{\xi_{2}}^{H}$ - $b h\left(\xi_{2}\right) c h y$ and $c h$ is a monomorphism. Thus

$$
(1+x) \rho^{k}\left(\xi_{1}\right)-k^{n_{1}-n_{2}} \rho^{k}\left(\xi_{2}\right) \psi^{k}(1+x)=0
$$

and the proposition follows.

Note that the condition that $K F(X)$ be torsion free in (2.3) is not necessary. It would suffice to ask that under some canonical splitting (e.g. filtration) $\rho^{k}\left(\xi_{1}\right)$ lie in the part of $K F(X)$, on which $c h$ is a monomorphism.

When dealing with spaces whose $K F$-theory is torsion free it is convenient to extend the character monomorphism ch: $K F(X) \rightarrow H^{*}(X ; Q)$ to $K F(X)$ $\otimes Q$. The resulting homomorphism is an isomorphism onto $H^{\mathrm{ev}}(X ; Q)$ when $F=U$ and onto $\sum_{i} H^{4 i}(X ; Q)$ when $F=O$. The inverse of this isomorphism will be denoted by $c h^{-1}$.

We introduce now a new characteristic class $B h(\xi) \in K F(X) \otimes Q$ as follows: 


$$
c h^{-1} U^{H}=U^{F} \cdot B H(\xi)^{-1} .
$$

This definition makes sense since $B h(\xi)$ is an element in the multiplicative group $1+(\widetilde{K F}(X) \otimes Q)$. Note that $B h$ (just as $\rho^{k}$ ) is multiplicative, i.e. $B h(\xi+\eta)=B h(\xi) B h(\eta)$ and since some multiple of any $F$-bundle is $K F$ orientable we can extend $B h$ over all of $K F(X)$ defining $B h(\xi)=[B h(m \xi)]^{1 / m}$. Applying the character to both sides of the defining relation for $B h(\xi)$ we find that

$$
B h(\xi)=c h^{-1} b h(\xi) .
$$

The following are useful corollaries of Proposition 2.3.

COROLlARY 2.6. If $K O(X)$ is torsion free then the KO-oriented bundles $\xi_{1}$ and $\xi_{2}$ are J-equivalent if and only if there exists $x \in \widetilde{K O}(X)$ such that bh$\left(\xi_{1}\right)$ $=b h\left(\xi_{2}\right) \operatorname{ch}(1+x)$, i.e. $B h\left(\xi_{1}\right)=(1+x) B h\left(\xi_{2}\right)$.

In particular, if $\xi_{2}$ is the trivial bundle then $b h\left(\xi_{2}\right)=1$ and we obtain

COROLLARY 2.7. If $K O(X)$ is torsion free then a bundle $\xi$ is $J$-trivial if and only if there exists $x \in \widetilde{K O}(X)$ such that $b h(\xi)=\operatorname{ch}(1+x)$, i.e. $B h(\xi)=(1+x)-$ an element in $1+\widetilde{K O}(X)$.

Another formulation of (2.7) is

COROLLARY 2.8. If $K O(X)$ is torsion free, then a bundle $\xi$ is J-trivial if and only if $U_{\xi}^{H}$ is in the image of the Chern character.

We should point out that the "integrality" of $B h(\xi)$ will not be used directly to compute the $J$-order of the Hopf bundle. The usefulness of the condition on $B h(\xi)$ will become apparent in the classification of stunted projective spaces.

We now turn our attention to the cannibalistic classes $\rho^{k}$. Let $\xi$ be a $K F$ orientable vector bundle of real cimension $2 n$. Consider the Chern character of $\rho^{k}(\xi)$.

$$
\begin{aligned}
\operatorname{ch} \psi^{k} U^{F} & =\bar{\psi}^{k} \operatorname{ch} U^{F}=\bar{\psi}^{k}\left(U^{H} b h(\xi)\right) \\
& =\bar{\psi}^{k} U^{H} \cdot \bar{\psi}^{k} b h(\xi)=k^{n} U^{H} \bar{\psi}^{k} b h(\xi) .
\end{aligned}
$$

Since $\operatorname{ch}\left(U^{F} \rho^{k}(\xi)\right)=U^{H} b h(\xi) \operatorname{ch} \rho^{k}(\xi)$, we obtain

$$
\operatorname{chp}^{k}(\xi)=k^{n} \bar{\psi}^{k} b h(\xi) / b h(\xi) .
$$

In particular, if $K F(X)$ is torsion free then

$$
\rho^{k}(\xi)=k^{n} \psi^{k} B h(\xi) / B h(\xi)
$$


COROLlARY 2.10. If $\xi$ is a real oriented vector bundle of dimension $2 n$ such that for some $k \neq 1$ we have

$$
\rho^{k}(\xi)=k^{n} \psi^{k}(1+x) /(1+x)
$$

for $x \in \widetilde{K O}(X)$ then $b h(\xi)=\operatorname{ch}(1+x)$. In particular, if $K O(X)$ is torsion free, the bundle $\xi$ is $J$-trivial.

Proof. Applying the Chern character to both sides of the equality we obtain

$$
\bar{\psi}^{k} b h(\xi) / b h(\xi)=\bar{\psi}^{k} \operatorname{ch}(1+x) / \operatorname{ch}(1+x)
$$

and the corollary follows from

LEMMA 2.11. The transformation $z \rightarrow \bar{\psi}^{k} z / z$ on the group $1+\tilde{H}^{\mathrm{ev}}(X ; Q)$ is an automorphism for all $k \neq 1$.

Proof. The transformation in question is clearly a group homomorphism. Let $z=1+\sum_{i>0} x_{i}$ with $x_{i} \in H^{2 i}(X ; Q)$. Then

$$
\bar{\psi}^{k} z=1+\sum k^{i} x_{i}
$$

and if $\bar{\psi}^{k} z / z=1$ then $1+\sum k^{i} x_{i}=1+\sum x_{i}$ and because $k \neq 1$, we obtain $x_{i}=0$ for all $i$. The proof that $z \rightarrow \bar{\psi}^{k} z / z$ is an epimorphism is just as simple.

Note that the "conjugate" homomorphism $x \rightarrow \psi^{k} x / x$ on $1+\widetilde{K F}(X)$ is also an automorphism for $k \neq 1$ whenever $K F(X)$ is torsion free. )

Proposition 2.12. Let $K F(X)$ be torsion free. Then $z \in \widetilde{K F}(X) \otimes Q$ is integral if and only if $\psi^{p}(1+z) /(1+z)$ is integral, for every prime $p$.

Proof. It suffices to prove that if $z \notin \widetilde{K F}(X)$ and $p z \in \widetilde{K F}(X)$ then $\psi^{p}(1+z) /(1+z)$ is not integral. We can also assume that $z+x$, where $x$ $\in \widetilde{K F}(X) \otimes Q$ is of higher filtration than $z$, is not integral for any such $x$.

First consider the case $F=U$. Since $z \in \widetilde{K U}(X) \otimes Q$ it has filtration $k \geqslant 1$. Now $\psi^{p}$ preserves filtration and $\psi^{p_{z}}=p^{k}{ }_{z}+a$ where $a$ is an element of higher filtration. We have

$$
\begin{aligned}
\psi^{p}(1+z) /(1+z) & =\left(1+p^{k} z+a\right)\left(1-z+z^{2}-\cdots\right) \\
& =1+\left(p^{k}-1\right) z+\text { elts of higher filtration }
\end{aligned}
$$

and this shows that $\psi^{p}(1+z) /(1+z)$ is not integral, as was claimed. The case $F=O$ follows by the same argument since when $K O(X)$ is torsion free the formula for $\psi^{p} z$ still holds with $k=$ (filtration of $c z$ ) $\geqslant 1$.

3. The $J$-order of the Hopf bundles. Let $\mathbf{Q} P^{\infty}$ and $\mathbf{Q} P^{n}$ denote the infiniteand $n$-dimensional projective spaces over the quaternions. Let $\xi$ denote the Hopf bundle over $\mathbf{Q} P^{\infty}$ as well as its restrictions to $\mathbf{Q} P^{n} \subset \mathbf{Q} P^{\infty}$. 
Recall (e.g. [14]) that $K U\left(\mathbf{Q} P^{n}\right)=Z[\mu] /\left(\mu^{n+1}\right)$ where $\mu=\xi-2$ and $c$ : $K O\left(\mathbf{Q} P^{n}\right) \rightarrow K U\left(\mathbf{Q} P^{n}\right)$ is a monomorphism whose image is the subring generated by $2 \mu$ and $\mu^{2}$. The complexification of the real vector bundle $\xi$ is $2 \mu+4$. The ring $K U\left(Q P^{\infty}\right)$ can be thought of as the ring of power series in $\mu$, and $K O\left(Q P^{\infty}\right)$ as the corresponding subring generated by $2 \mu$ and $\mu^{2}$.

We choose $y \in H^{4}\left(Q P^{\infty}, Z\right)$ so that $1, y, y^{2}, \ldots$ gives a basis for $H^{*}\left(\mathbf{Q} P^{\infty}\right)$ and $H^{*}\left(\mathbf{Q} P^{n}\right)$ in such a way that $y \in H^{4}\left(\mathbf{Q} P^{n}\right)$ is the characteristic class of the normal bundle of the natural inclusion $Q P^{n} \subset Q P^{n+1}$ i.e. the Hopf bundle $\xi$ over $\mathbf{Q} P^{n}$. We denote by $y$ the fundamental class in $H^{*}\left(\mathbf{Q} P^{\infty}\right)$ as well as its restrictions to $H^{*}\left(\mathbf{Q} P^{n}\right)$.

We have short exact sequences $(k \leqslant n)$

$$
0 \rightarrow H^{*}\left(\mathbf{Q} P_{k}^{n}\right) \stackrel{j^{*}}{\longrightarrow} H^{*}\left(\mathbf{Q} P^{n}\right) \stackrel{i^{*}}{\longrightarrow} H^{*}\left(\mathbf{Q} P^{k-1}\right) \rightarrow 0
$$

and

$$
0 \rightarrow K U\left(\mathbf{Q} P_{k}^{n}\right) \stackrel{j^{!}}{\rightarrow} K U\left(\mathbf{Q} P^{n}\right) \stackrel{i !}{\longrightarrow} K U\left(\mathbf{Q} P^{k-1}\right) \rightarrow 0
$$

corresponding to the cofibration

$$
\mathbf{Q} P^{k-1} \rightarrow \mathbf{Q} P^{n} \rightarrow \mathbf{Q} P_{k}^{n} .
$$

Identifying $\tilde{H}^{*}\left(\mathrm{Q} P_{k}^{n}\right)$ with $\operatorname{ker} j^{*}$ we obtain the basis $y^{k}, y^{k+1}, \ldots$, $y^{n}$ for $\tilde{H}^{*}\left(\mathbf{Q} P_{k}^{n}\right)$ and similarly $\mu^{k}, \ldots, \mu^{n}$ as basis for $\widetilde{K U}\left(\mathbf{Q} P_{k}^{n}\right)$. Note that $\Lambda_{t} \mu^{t}$ with $\Lambda_{t}=1$ if $t$ is even and $\Lambda_{t}=2$ if $t$ is odd give a basis for $c K O\left(Q P_{k}^{n}\right)$.

We also have the identification $Q P_{k}^{m+k} \simeq\left(Q P^{m}\right)^{k \xi}$ and a basis for $\tilde{H}^{*}\left(\mathbf{Q} P_{k}^{m+k}\right)$ via the Thom isomorphism. One easily checks that, with our choice of $y$, these two bases coincide. The same holds true for $K U\left(\mathbf{Q} P_{k}^{m+k}\right)$. Also for $K O\left(\mathbf{Q} P_{k}^{m+k}\right)$ whenever $k$ is even.

Note that $U_{k \xi}^{H}=y^{k}, U_{k \xi}^{U}=\mu^{k}=c U_{k \xi}^{O}$ for $k$ even. Since

$$
c^{-1} c h^{-1} U_{k \xi}^{H}=U_{k \xi}^{O} \cdot B h_{O}(-k \xi) \text { and } c h^{-1} U_{k \xi}^{H}=U_{k \xi}^{U} \cdot B h_{U}(-k \xi),
$$

we see that $c B h_{O}(-k \xi)=B h_{U}(-k \xi)$.

In what follows we shall identify $\widetilde{K O}\left(Q P^{n}\right)$ with its complexification and consider it a subring of $\widetilde{K U}\left(\mathbf{Q} P^{n}\right)$. Note that

$$
K O\left(Q P^{n}\right) \otimes Q \stackrel{C \otimes 1}{\longrightarrow} K U\left(Q P^{n}\right) \otimes Q
$$

is an isomorphism. With this convention, $B h_{O}(\xi)=B h_{U}(\xi)$, and we will denote it simply by $B h(\xi)$.

Proposition 3.1. In $\mathbf{Q} P^{n}$ the following statements are equivalent.

(i) The bundle $k \xi$ is $J$-trivial.

(ii) The class $B h(k \xi)$ belongs to $1+\widetilde{K O}\left(\mathbf{Q} P^{n}\right)$. 
(iii) The class $\psi^{p} B h(k \xi) / B h(k \xi)$ belongs to $1+\widetilde{K O}\left(Q P^{n}\right)$ for all primes $p$.

(iv) The class $\left(\psi^{p} \mu / p^{2} \mu\right)^{k}$ belongs to $1+\widetilde{K O}\left(Q P^{n}\right)$ for all primes $p$.

Proof. Proposition (2.5) shows that (i) and (ii) are equivalent. Proposition (2.12) shows that (ii) and (iii) are equivalent. Now from (2.9), we have

$$
\rho^{p}(2 \xi)=p^{4} \psi^{p} B h(2 \xi) / B h(2 \xi) .
$$

Also

$$
\psi^{p} U_{2 \xi}^{0}=U_{2 \xi}^{0} \cdot \rho^{p}(2 \xi),
$$

hence $\rho^{p}(2 \xi)=\psi^{p} U_{2 \xi}^{0} / U_{2 \xi}^{0}$ and identifying $\rho^{p}(2 \xi)$ with its image in $K U\left(\mathbf{Q} P^{n}\right)$, we have

$$
\rho^{p}(2 \xi)=\left(\psi^{p} \mu / \mu\right)^{2} .
$$

From (1) and (2) we obtain

$$
\psi^{p} B h(2 \xi) / B h(2 \xi)=\left(\psi^{p} \mu / p^{2} \mu\right)^{2}
$$

and hence (note that $k$ must be even)

$$
\psi^{p} B h(k \xi) / B h(k \xi)=\left(\psi^{p} \mu / p^{2} \mu\right)^{k} ;
$$

thus (iii) and (iv) are equivalent and (3.1) follows.

In [7] we gave the following explicit formula for $\psi^{p}$ in $\mathbf{Q} P^{\infty}$ :

$$
\psi^{p}(\mu)=\sum_{m=1}^{p}\left(\begin{array}{c}
p+m-1 \\
2 m-1
\end{array}\right) \frac{p}{m} \mu^{m} .
$$

We now will show the following:

Proposition 3.3. Let $\mu=\xi-2$ denote the generator in $K U\left(\mathbf{Q} P^{\infty}\right)$. Then $\psi^{2} \mu / \mu=4+\mu$ and for $p$ odd

$$
\psi^{p}(\mu) / \mu=\left(p \lambda+\mu^{(p-1) / 2}\right)^{2}
$$

where $\lambda \in 1+\widetilde{K U}\left(\mathbf{Q} P^{\infty}\right)$.

PROoF. We begin by showing that $\psi^{P}(\mu) / \mu$ is a square whenever $p$ is odd. Consider the standard fibration $\pi: \mathbf{C} P^{\infty} \rightarrow \mathbf{Q} P^{\infty}$. Then $\pi^{*} \mu=\xi+\xi^{-1}-2$ where $\xi$ is the Hopf bundle over $\mathbf{C} P^{\infty}$ and

$$
\psi^{p} \pi^{*}(\mu)=\xi^{p}+\xi^{-p}-2=\left(\xi^{p}-1\right)^{2} / \xi^{p} .
$$

Since $\pi^{*}$ is a monomorphism we find $\psi^{p}(\mu)$ by expressing $x^{p}+x^{-p}-2$ as a polynomial in $x+x^{-1}-2$ and setting $\mu=x+x^{-1}-2$. Now $\alpha+\alpha^{-1}-2$ is 
a root of that polynomial, whenever $\alpha$ is a root of $x^{p}-1$. The roots of $x^{p}-1$ are $e^{2 \pi i s / p}$ for $0 \leqslant s \leqslant p-1$ and so the roots of $\psi^{p}(\mu)$ as a polynomial in $\mu$ are $e^{2 \pi i s / p}+e^{2 \pi i(p-s) / p}-2$. If $p$ is odd, one of the roots $(s=0)$ is zero and all others are double roots. Thus $\psi^{p}(\mu)=\mu[R(\mu)]^{2}$ and $R(\mu)$ is an integral polynomial since $\psi^{p}(\mu) / \mu$ is. It follows from (3.2) that the coefficient of $\mu^{m}$ in $[R(\mu)]^{2}$ for $m<(p+1) / 2$ is divisible by $p^{2}$ and so $R(\mu)=p \lambda$ $+\mu^{(p-1) / 2}$ as was claimed. The formula for $\psi^{2}(\mu) / \mu$ is also contained in (3.2).

We are now ready to prove:

THEOREM 3.4. The J-order $B_{n}$ of the Hopf bundle over $\mathbf{Q} P^{n}$ is given by

$$
B_{n}=\prod p^{v_{p}\left(B_{n}\right)}
$$

where

$$
\nu_{2}\left(B_{n}\right)=\max \left\{2 n+1,2 j+\nu_{2}(j) \mid 1 \leqslant j \leqslant n\right\}
$$

and

$$
\nu_{p}\left(B_{n}\right)=\max \left\{j+\nu_{p}(j) \mid 1 \leqslant j \leqslant 2 n /(p-1)\right\}
$$

when $p$ is an odd prime.

Proof. By Stiefel-Whitney class consideration $B_{n}$ is even, so that we may apply (3.1). Therefore it suffices to prove that $B_{n}$ is the least integer $s$ for which

$$
\left(\psi^{p} \mu / p^{2} \mu\right)^{s} \in 1+\widetilde{K O}\left(\mathbf{Q} P^{n}\right)
$$

for all primes $p$.

We need a lemma on divisibility of binomial coefficients.

LemMa 3.6. Let $p$ be a prime and suppose $p^{j}$ divides $\left(\begin{array}{l}s \\ j\end{array}\right)$ for all $1 \leqslant j \leqslant q$. Then $\nu_{p}(s)-\nu_{p}(j)=\nu_{p}\left(\begin{array}{l}s \\ j\end{array}\right)$ for $1 \leqslant j \leqslant q$.

Assuming (3.6) for the moment, consider the case $p=2$. Then we need to see that $\left(\psi^{2} \mu / p^{2} \mu\right)=(1+\mu / 4)^{s}$ lies in $1+\widetilde{K O}\left(Q P^{n}\right)$. This requires that $2^{2 j}$ divide $\left(\begin{array}{l}s \\ j\end{array}\right)$ if $j$ is even, and $2^{2 j+1}$ divide $\left(\begin{array}{l}s \\ j\end{array}\right)$ if $j$ is odd, for all $1 \leqslant j \leqslant n$. Applying (3.6) we obtain $\nu_{2}(s)-\nu_{2}(j) \geqslant 2 j$ if $j$ is even and $\nu_{2}(s) \geqslant 2 j+1$ if $j$ is odd. Thus the expression for $\nu_{2}\left(B_{n}\right)$.

Now consider the case $p$ odd. Then, by (3.3) we obtain that (3.5) is given as follows

$$
\left(\psi^{p} \mu / p^{2} \mu\right)^{s}=\left(\lambda+p^{-1} \mu^{(p-1) / 2}\right)^{2 s}, \quad \lambda \in 1+\widetilde{K U}\left(\mathbf{Q} P^{n}\right) .
$$

Notice that $1+x$ belongs to $1+\widetilde{K U}\left(Q P^{n}\right)$, if and only if $(1+x)^{2}$ belongs to $1+\widetilde{K O}\left(\mathbf{Q} P^{n}\right)$. Therefore it suffices to see that $\left(\lambda+p^{-1} \mu^{(p-1) / 2}\right)^{s}$ belongs to $1+\widetilde{K U}\left(\mathbf{Q} P^{n}\right)$. This requires that $p^{j}$ divide $\left(\begin{array}{l}s \\ j\end{array}\right)$ for all $1 \leqslant j \leqslant 2 n /(p-1)$. By 
(3.6), we obtain $\nu_{p}\left(\begin{array}{l}s \\ j\end{array}\right)=\nu_{p}(s)-\nu_{p}(j) \geqslant j, 1 \leqslant j \leqslant 2 n /(p-1)$, and this gives the expression for $\nu_{p}\left(B_{n}\right)$.

PROOF OF (3.6). We prove it by induction on $q$. It is true for $q=1$, since $\nu_{p}\left(\begin{array}{l}s \\ 1\end{array}\right) \geqslant 1$ implies $s \equiv O(p)$ and $\nu_{p}(1)=0$, so $v_{p}\left(\begin{array}{l}s \\ 1\end{array}\right)=v_{p}(s)$. Assume the result true for $q-1$. Then we consider two cases.

Case 1. $q \not \equiv 1(p)$. We have

$$
\left(\begin{array}{l}
s \\
q
\end{array}\right)=\frac{s(s-1) \cdots(s-q+2)}{1 \cdots(q-1)} \frac{(s-q+1)}{q}
$$

and

$$
\nu_{p}\left(\begin{array}{c}
s \\
q-1
\end{array}\right)=\nu_{p}(s)-\nu_{p}(q-1)=\nu_{p}(s) .
$$

Now $(s-q+1) \neq \equiv 0(p)$. Hence $\nu_{p}\left(\begin{array}{l}s \\ q\end{array}\right)=\nu_{p}(s)-\nu_{p}(q)$.

Case 2. $q \equiv 1(p)$. Then

$$
\nu_{p}\left(\begin{array}{c}
s \\
q-1
\end{array}\right)=\nu_{p}(s)-\nu_{p}(q-1)
$$

and

$$
\nu_{p}\left(\begin{array}{l}
s \\
q
\end{array}\right)=\nu_{p}(s)-\nu_{p}(q-1)+\nu_{p}(s-q+1)-\nu_{p}(q) .
$$

But $\nu_{p}(s) \geqslant \nu(q-1)+q-1$, by hypothesis, hence $\nu_{p}(s-q+1)=p_{p}(q-1)$, and we have $v_{p}\left(\begin{array}{l}s \\ q\end{array}\right)=v_{p}(s)$. This ends the proof of (3.6).

Note that K. Y. Lam in [12] used a similar number-theoretical argument to obtain the Atiyah-Todd number $M_{n+1}$, which was proven by Adams and Walker in [3] to be the $J$-order of the Hopf bundle over $\mathbf{C} P^{n}$. We offer here another proof of this fact.

DEFINITION 3.5. Let $I(n)$ denote the ring of those power series $a_{0}+a_{1} z+\cdots$ $+a_{k} z^{k}+\cdots$ in $Q[[z]]$ such that $a_{i} \in Z$ for $i \leqslant n$. Let $I_{2}(n)$ denote the subring of $I(n)$ for which $a_{j} \in 2 Z$ if $j$ is odd and $j \leqslant n$.

Proposition 3.6. The J-order $A_{n}$ of the Hopf bundle over $\mathbf{C} P^{n}$ is equal to the Atiyah-Todd number $M_{n+1}$.

Proof. Recall from [4] that the Atiyah-Todd number $M_{n+1}$ was defined as the least number $s$ such that the power series:

$$
L^{s}(z)=[\ln (1+z) / z]^{s}
$$

belongs to $I(n)$.

Recall from Sanderson in [14], that 


$$
\begin{aligned}
K O\left(\mathbf{C} P^{n}\right) & = \begin{cases}Z[\alpha] /\left(\alpha^{[n / 2]+1}\right) & \text { if } n \neq 1(4), \\
Z[\alpha] /\left(2 \alpha^{[n / 2]+1}, \alpha^{[n / 2]+2}\right) & \text { if } n \equiv 1(4),\end{cases} \\
K U\left(\mathbf{C} P^{n}\right) & =Z[\beta] /\left(\beta^{n+1}\right)
\end{aligned}
$$

and

$$
H^{*}\left(\mathbf{C} P^{n} ; Z\right)=Z[x] /\left(x^{n+1}\right) .
$$

The generators are $\alpha=r(\xi)-2, \beta=\xi-1, x=c_{1}(\xi)$, where as above $\xi$ denotes the Hopf bundle over $\mathbf{C} P^{n}$. If $c$ is the complexification,

$$
c=\beta^{2} /(1+\beta) \text {. }
$$

Also $\operatorname{ch} \beta=e^{x}-1$, so that $c^{-1}(x)=\ln (1+\beta)$.

Since $4 \xi$ is $K O$-orientable over $\mathrm{C} P^{n}$ and $\alpha^{2} \in K O\left(\mathrm{CP}_{4}^{n+4}\right)$, is its Thom class, we have

$$
\operatorname{ch}\left(c \alpha^{2}\right)=x^{4} \cdot b h(4 \xi)
$$

and

$$
c h^{-1}\left(x^{4}\right)=c \alpha^{2} \cdot c B h(-4 \xi)
$$

hence

$$
(\ln (1+\beta))^{4}=\frac{\beta^{4}}{(1+\beta)^{2}} c B h(-4 \xi)
$$

Therefore

$$
c B h(\xi)=\frac{\beta}{\sqrt{1+\beta} \ln (1+\beta)} .
$$

On the other hand $\rho^{k}(4 \xi)=\psi^{k}\left(\alpha^{2}\right) / \alpha^{2}$. Now, in $\mathbf{C} P^{n+4}, \psi^{k} \alpha=k^{2} \alpha+\cdots$ $+\alpha^{[n / 2]+2}+\varepsilon \alpha^{(n+5) / 2}$ with possibly $\varepsilon \neq 0$ when $n \equiv 1$ (4). Therefore $\psi^{k}\left(\alpha^{2}\right)$ $=k^{4} \alpha^{2}+\cdots+a \alpha^{[n / 2]+2}$ and $\rho^{k}(4 \xi)$ as well as $\rho^{k}(4 m \xi)$ have only terms $a_{i} \alpha^{i}$ for $i<(n+1) / 2$. Since this is a "canonical" torsion free summand of $K O\left(C P^{n}\right)$, by the note after Corollary 2.5 it suffices that $B h(4 m \xi)$ be integral for $4 m \xi$ to be $J$-trivial. Since $c: \alpha \rightarrow \beta^{2}-\beta^{3}+\cdots$ has leading coefficient 1 it indeed suffices that $c B h(4 m \xi)$ be integral and since $1+\beta$ is invertible in $1+\widetilde{K U}\left(C P^{n}\right)$ the claim follows.

REMARK. Consider the diagram:

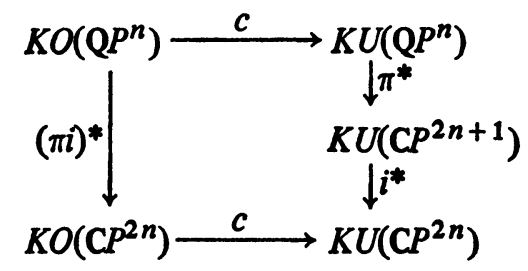


Here $c$ and $(\pi i)^{*}$ are monomorphisms. Moreover

$$
c\left(K O\left(\mathbf{C} P^{2 n}\right)\right)=(\pi i)^{*} K U\left(\mathbf{Q} P^{n}\right)
$$

thus $\varphi: K U\left(\mathbf{Q} P^{n}\right) \rightarrow K O\left(\mathbf{C} P^{2 n}\right)$ given by $\varphi(\mu)=\alpha$ is an isomorphism of $\psi$ rings and we could have computed $B_{n}$ from our knowledge of $A_{2 n}$. Namely, we have: $v_{p}\left(B_{n}\right)=v_{p}\left(A_{2 n}\right)$ for $p$ odd. This follows from the fact that $\varphi B h\left(\xi_{Q}\right)$ $=B h\left(2 \xi_{c}\right)=L(\alpha)$ and that from (3.6) and (3.7) we have

$$
2 k \xi_{c} \text { is } J \text {-trivial if and only if } L^{k}(x) \in I(n)
$$

and

$$
k \xi_{Q} \text { is } J \text {-trivial if and only if } L^{k}(x) \in I_{2}(n) .
$$

To complete the computation of $B_{n}$ we would only need to use

$$
\psi^{2}(\mu)=4 \mu+\mu^{2}
$$

4. Homotopy equivalences and their representations in ordinary cohomology and $K$-theory. The object of this section is to determine the homomorphisms $f^{!}: K U\left(\mathbf{Q} P_{k}^{n+k}\right) \rightarrow K U\left(\mathbf{Q} P_{l}^{n+l}\right)$ induced by the possible stable homotopy equivalences between the two spaces. This will provide the vital necessary conditions in Theorem 1.2. The main tool here is the commutativity of the diagram

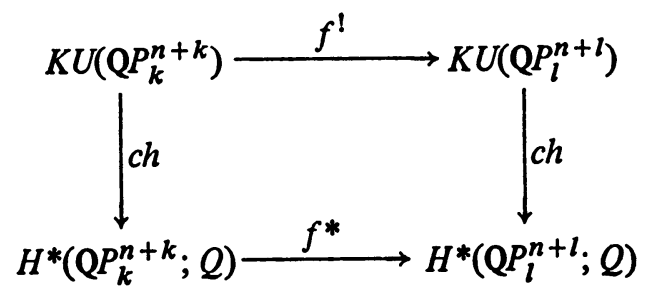

for a stable homotopy equivalence $f: \mathbf{Q} P_{l}^{n+l} \rightarrow \mathbf{Q} P_{k}^{n+k}$. Note that $f^{*}$ and $f^{!}$ are taken in conjunction with suspension isomorphism and Bott periodicity. We shall also use the fact that $f^{!}$must preserve both $K O\left(\mathbf{Q} P_{r}^{t}\right)$ and $K \operatorname{Sp}\left(\mathbf{Q} P_{r}^{t}\right)$ embedded in $K U\left(\mathbf{Q} P_{r}^{t}\right)$. This fact implies that $k \equiv l(2)$ for a possible equivalence. Here the groups are as defined in $\$ 3$ and we will continue to use the notation there introduced.

First observe that $f^{*}$ can be thought of as a diagonal $(n+1) \times(n$ $+1)$ matrix $\left(\varepsilon_{0}, \varepsilon_{1}, \ldots, \varepsilon_{n}\right)$ with $\varepsilon_{i}= \pm 1$. Since each of the spaces in question admits a self-equivalence which induces the matrix $(-1,-1, \ldots,-1)$ in cohomology, the matrices $\left(\varepsilon_{i}\right)$ and $\left(-\varepsilon_{i}\right)$ can be thought of as equivalent.

Since $c h$ is a rational isomorphism, $f^{!}: K U\left(\mathbf{Q} P_{k}^{n+k}\right) \otimes Q \rightarrow K U\left(\mathbf{Q} P_{l}^{n+l}\right)$ 
$\otimes Q$ is uniquely determined by $f^{*}$; namely $f^{!}=c h^{-1} f^{*} c h$. Thus $f^{!}$is, in some sense, an adjoint of $f^{*}$. Since $f^{!}$must be integral we obtain restrictions on the possible homomorphisms $f^{*}$.

Let us compute $f^{!}$on $\mu^{k}$ when $f^{*}$ is the identity matrix $I$. We have $f^{!}\left(\mu^{k}\right)=c h^{-1} I c h \mu^{k}$. Recall that $\mu^{k}=U_{k \xi}^{U}$, hence $c h \mu^{k}=U_{k \xi}^{H} b h_{c}(k \xi)$. Now $f^{*}$ being the "identity" replaces $U_{k \xi}^{H}$ by $U_{l \xi}^{H}$ and so

$$
f^{!}\left(\mu^{k}\right)=c h^{-1}\left(U_{l \xi}^{H} b h(k \xi)\right) .
$$

We have, by (2.5) and (2.6),

$$
f^{\prime}\left(\mu^{k}\right)=U_{l \xi}^{U} \cdot B h_{c}(-l \xi) \cdot B h_{c}(k \xi)=\mu^{l} \cdot B h_{c}((k-l) \xi) .
$$

From $K U$ considerations alone, we conclude that $B h_{c}((k-l) \xi)$ must be integral.

We now determine the class $B h_{c}(\xi)$ as a power series. Recall that chu $=e^{x}+e^{-x}-2$, with $y=x^{2}$. Thus

$$
\operatorname{ch} \mu=y+2 y^{2} / 4 !+2 y^{3} / 6 !+\cdots .
$$

Thus chu is a power series which begins with $y$ and hence it can be inverted (in the sense of substitution of series) to obtain

$$
c h^{-1} y=\mu+a_{2} \mu^{2}+\cdots \text { and } B h(-\xi)=1+\sum a_{i} \mu^{i} .
$$

We will denote the power series $1+\sum_{r>0} a_{r} x^{r}$ by $t(X)$. It is elementary to determine the coefficients $a_{r}$, which we include here for the sake of completeness:

$$
a_{r}^{-1}=(-1)^{r}(r+1)(2 r+1)\left(\begin{array}{c}
2 r \\
r
\end{array}\right) \text {. }
$$

Now $f^{!}$must preserve both $K O$ and $K S$. Moreover $K F\left(\mathbf{Q} P_{k}^{n+k}\right)$ $\subset K U\left(\mathbf{Q} P_{k}^{n+k}\right)$ is generated by $\mu^{k}, 2 \mu^{k+1}, \mu^{k+2}, \ldots$ if $F=\mathrm{Sp}$ and $k$ is odd or if $F=O$ and $k$ is even. It follows that $B h_{c}((k-l) \xi)=(T(\mu))^{k-l}$ not only belongs to $I(n)$ but also to $I_{2}(n)$.

We obtain:

Proposition 4.1. There exists a stable homotopy equivalence $f: \mathbf{Q} P_{l}^{n+l}$ $\rightarrow \mathbf{Q} P_{k}^{n+k}$ with $f^{*}=(1,1, \ldots, 1)$ if and only if $k=l\left(B_{n}\right)$. In this case $f^{!} \mu^{k+i}$ $=\mu^{l+i} \cdot(T(\mu))^{l-k}$.

Note: In [11], Held and Sutter have proved general results which include (4.1), as a special case.

Our next aim is to prove that, save multiplication by $-I$, the only possible matrices which can occur as $f^{*}$ are $(1,1, \ldots, 1)$ and $(-1,1,1, \ldots, 1)$. Since an 
equivalence $f$ can always be made cellular, it suffices to prove our assertion for $3 \times 3$ matrices, i.e. for possible equivalences between Thom spaces over $Q P^{2}$. To this end we first prove:

LEMMA 4.2. Let $f: \mathbf{Q} P_{l}^{l+1} \rightarrow \mathbf{Q} P_{k}^{k+1}$ be a stable homotopy equivalence such that $f^{*}=(-1,1)$. Then $k+l \equiv 0(24)$.

Proof. As before, we compute $f^{!}\left(\mu^{k}\right)$. We have

$$
f^{!} \mu^{k}=c h^{-1} f^{*} c h \mu^{k}=c h^{-1} f^{*}\left(U_{k \xi}^{H} b h(k \xi)\right) .
$$

But $f^{*}=(-1,1)$, hence

$$
\begin{aligned}
f^{!} \mu^{k} & =c h^{-1}\left(U_{l \xi}^{H}(b h(k \xi)-2)\right) \\
& =-U_{l \xi}^{U} \cdot B h(-l \xi)(2-B h(k \xi)) \\
& =-U_{l \xi}^{U} \cdot(B h((k-l) \xi)-2 B h(-l \xi)) \\
& =-\mu^{l} \cdot(B h(k-l) \xi)(2 B h(-k \xi)-1) .
\end{aligned}
$$

Hence

$$
f^{!} \mu^{k}=-\mu^{l} \cdot T^{l-k}(\mu)\left(2 T^{k}(\mu)-1\right)
$$

where we have set $T^{r}(\mu)=(T(\mu))^{r}$. Our requirement now is

$$
T^{l-k}(x)\left(2 T^{k}(x)-1\right) \in I_{2}(1) .
$$

But $T(x)=1-x / 12+x^{2} / 90+\cdots$, we have

$$
\left(1-\frac{x}{12}\right)^{l-k}\left(2\left(1-\frac{x}{12}\right)^{k}-1\right)=1-\frac{l+k}{12} x+\cdots \in I_{2}(1)
$$

and so $l+k \equiv 0(24)$ as was claimed.

REMARK. The formula (4.3) holds generally for equivalences $f$ such that $f^{*}=(-1,1,1, \ldots, 1)$.

LEMMA 4.4. Let $f: \mathbf{Q} P_{l}^{l+2} \rightarrow \mathbf{Q} P_{k}^{k+2}$ be a stable homotopy equivalence. Then, up to multiplication by $-I$, either $f^{*}=(1,1,1)$ or $f^{*}=(-1,1,1)$.

Proof. It suffices to rule out the possibilities $(1,-1,1)$ and $(1,1,-1)$ for $f^{*}$. We assume that $f$ is cellular. By restriction to $f_{1}: \mathbf{Q} P_{l}^{l+1} \rightarrow \mathbf{Q} P_{k}^{k+1}$ we find, in the case $(1,-1,1)$, that $k+l \equiv 0(24)$. On the other hand, we also have an equivalence on the coskeleta: $f_{2}: \mathbf{Q} P_{l+1}^{l+2} \rightarrow \mathbf{Q} P_{k+1}^{k+2}$ with $f_{2}^{*}=(-1,1)$ and so $k+l+2 \equiv 0$ (24) which contradicts the first congruence. This rules out the case $f^{*}=(1,-1,1)$. For the case $f^{*}=(1,1,-1)$ we compute $f^{!}\left(\mu^{k}\right)$. We have 


$$
\begin{aligned}
f^{*} \operatorname{ch} \mu^{k} & =f^{*}\left[y^{k} b h(k \xi)\right]=f^{*}\left[y^{k}\left(1+\frac{2 y}{4 !}+\frac{2 y^{2}}{6 !}\right)^{k}\right] \\
& =f^{*}\left[y^{k}\left(1+\frac{2 k}{4 !} y+\frac{k(5 k-1)}{1440} y^{2}\right)\right] \\
& =y^{l}\left[b h(k \xi)-\frac{k(5 k-1)}{720} y^{2}\right] .
\end{aligned}
$$

Here $y^{l}=U_{l \xi}^{H}$ and since $c h^{-1} y^{l+2}=\mu^{l+2}=U_{l \xi}^{U} \cdot \mu^{2}$ we obtain

$$
\begin{aligned}
f^{!} \mu^{k} & =U_{l \xi}^{U} \cdot B h(-l \xi)\left[B h(k \xi)-\frac{k(5 k-1)}{720} \mu^{2}\right] \\
& =U_{l \xi}^{U} \cdot B h((k-l) \xi)\left[1-B h(-k \xi) \frac{k(5 k-1)}{720} \mu^{2}\right] \\
& =\mu^{l} \cdot B h((k-l) \xi)\left[1-\frac{k(5 k-1)}{720} \mu^{2}\right] .
\end{aligned}
$$

This last equality holds because we are in $K U\left(\mathbf{Q} P_{l}^{l+2}\right)$. Our integrality condition imposes then

$$
\left(1-\frac{k(5 k-1)}{720} x^{2}\right)\left(1-\frac{x}{12}+\frac{x^{2}}{90}\right)^{1-k} \in I_{2}(2) .
$$

This yields $l-k \equiv 0(24)$, since the coefficient of $x$ is $(l-k) / 12$. One finds the coefficient of $x^{2}$ to be

$$
\left(\begin{array}{c}
l-k \\
2
\end{array}\right) \frac{1}{12^{2}}+\frac{l-k}{90}-\frac{k(5 k-1)}{720}
$$

and since this coefficient must be an integer we obtain the condition

$$
5(l-k)(l-k-1)+2^{4}(l-k)-2 k(5 k-1) \equiv 0(24 \times 60) .
$$

Calculating modulo 24 , since $l-k=0$ (24), we obtain

$$
k(5 k-1) \equiv 0(12)
$$

On the other hand the map on the coskeleta

$$
f_{2}: \mathbf{Q} P_{l+1}^{l+2} \rightarrow \mathbf{Q} P_{k+1}^{k+2}
$$

induced by $f$ yields $f_{2}^{*}=(1,-1)$ which, by Lemma 4.2 , implies $k+l+2$ $\equiv 0(24)$. This together with $k-l \equiv 0(24)$ yields

$$
k \equiv-1(12) \text { and } k(5 k-1) \equiv 6(12)
$$

This, however, is in contradiction with the congruence (4.5). 
Corollary 4.6. Let $f: \mathbf{Q} P_{l}^{n+l} \rightarrow \mathbf{Q} P_{k}^{n+k}$ be a stable homotopy equivalence. Then, up to multiplication by $-I$, either $f^{*}=(1,1, \ldots, 1)$ or $f^{*}=(-1,1, \ldots$, 1).

We now proceed to study $f^{!}$when $f^{*}$ is of the form $(-1,1, \ldots, 1)$. By $(4.3)$ we have

$$
T^{l-k}(x)\left[2 T^{k}(x)-1\right] \in I_{2}(n) .
$$

Moreover, by considering the equivalence $f_{2}: \mathbf{Q} P_{l+1}^{n+l} \rightarrow \mathbf{Q} P_{k+1}^{n+k}$ induced by $f$ we have, by Proposition 4.1, $l-k \equiv O\left(B_{n-1}\right)$. We are now ready to prove

Proposirion 4.7. If $: \mathbf{Q} P_{l}^{n+l} \rightarrow \mathbf{Q} P_{k}^{n+k}$ is a stable homotopy equivalence with $f^{*}=(-1,1, \ldots, 1)$ then $k-l \equiv O\left(B_{n-1}\right)$ and $k+l \equiv O\left(B_{n}\right)$. Moreover

$$
f^{!}\left(\mu^{k}\right)=\mu^{l} T^{l-k}(\mu)\left[2 T^{k}(\mu)-1\right]
$$

and

$$
f^{!}\left(\mu^{k+i}\right)=\mu^{l+i} T^{l-k}(\mu)
$$

for $i>0$.

This proposition provides the desired necessary condition in Theorem 1.2. We first prove two lemmas. The first is a simple statement about the growth of $B_{n}$.

LEMMA 4.8. If $2 k \equiv O\left(B_{n-1}\right)$ and $n>2$ then $k \equiv O\left(B_{[n / 2]}\right)$.

This is a very weak statement which follows upon direct inspection of the formula for $\nu_{2}\left(B_{n}\right)$ and the inequality $\log _{2} n<n / 2$.

LEMMA 4.9. If $2 T^{k}(x)-1 \in I_{2}(n)$ then $T^{2 k}(x) \in I_{2}(n)$.

Proof. For $n=1$ the claim is obvious. Suppose that

$$
2 T^{k}(x)-1 \in I_{2}(n) \subset I_{2}(n-1) .
$$

We have, by induction, $T^{2 k}(x) \in I_{2}(n-1)$ and by Lemma (4.8)

$$
T^{k}(x) \in I_{2}([n / 2])
$$

i.e.

$$
T^{k}(x)=1+a_{1} x+\cdots+a_{[n / 2]} x^{[n / 2]}+\frac{1}{2}\left(a_{[n / 2]} x^{[n / 2]+1}+\cdots+a_{n} x^{n}\right)+\cdots
$$

where $a_{i}$ are integers which are even for $i$ odd, $i \leqslant n$.

Since $2+2[n / 2]>n$ we conclude that

$$
\left[T^{k}(x)\right]^{2}=T^{2 k}(x) \in I_{2}(n) .
$$


Proof of Proposition (4.7). For $n=1$ the statement is that of Lemma 4.2. Note that the formulas in Proposition (4.7) follow from (4.3) and Proposition (4.1) by collapsing the bottom cells.

It remains to prove that the condition

$$
T^{l-k}(x)\left[2 T^{k}(x)-1\right] \in I_{2}(n)
$$

together with $l-k \equiv O\left(B_{n-1}\right)$ implies $l+k \equiv O\left(B_{n}\right)$. Since $l-k$ $\equiv O\left(B_{n-1}\right)$ we have $T^{l-k} \in I_{2}(n-1)$ and hence

$$
2 T^{k}(x)-1 \in I_{2}(n-1) \text {. }
$$

By Lemma 4.8 this implies that $T^{2 k}(x) \in I_{2}(n-1)$ and, by Lemma (4.8), $T^{k}(x) \in I_{2}([n / 2])$ as long as $n>2$. We can write

$$
\begin{aligned}
T^{k}(x)= & 1+a_{1} x+\cdots+a_{[n / 2]} x^{[n / 2]} \\
& +\frac{1}{2}\left(a_{[n / 2]+1} x^{[n / 2]+1}+\cdots+a_{n-1} x^{n-1}+\alpha x^{n}+\cdots\right)
\end{aligned}
$$

and

$$
T^{l-k}(x)=1+b_{1} x+\cdots+b_{n-1} x^{n-1}+\beta x^{n}+\cdots
$$

where the $a_{i}$ 's and $b_{i}$ 's are integers which are even for $i$ odd and $\alpha, \beta$ are some rational numbers.

We have

$$
2 T^{k}(x)-1=1+\cdots+a_{n-1} x^{n-1}+2 \alpha x^{n}+\cdots
$$

and

$$
T^{2 k}(x)=1+\cdots+(\theta+2 \alpha) x^{n}+\cdots
$$

where $\theta$ is an integer which is even if $n$ is odd. This follows since

$$
2+2[n / 2]>n \text {. }
$$

Since

$$
T^{l-k}(x)\left[2 T^{k}(x)-1\right] \in I_{2}(n)
$$

$2 \alpha+\beta$ must be an integer of appropriate parity. This, clearly, implies that $T^{l-k}(x) \cdot T^{2 k}(x)=T^{l+k}(x) \in I_{2}(n)$ i.e. $l+k \equiv O\left(B_{n}\right)$.

It remains to verify the case $n=2$. This is a routine calculation with $T(x)=1-x / 12+x^{2} / 90+\cdots$.

We have 


$$
\begin{aligned}
T^{l-k}(x)[ & \left.2 T^{k}(x)-1\right] \\
= & \left(1-\frac{l-k}{12} x+\left[\left(\begin{array}{c}
l-k \\
2
\end{array}\right) \frac{1}{12^{2}}+\frac{l-k}{90}\right] x^{2}\right) \\
& \cdot\left(1-\frac{2 k}{12} x+\left[\left(\begin{array}{l}
k \\
2
\end{array}\right) \frac{2}{12^{2}}+\frac{2 k}{90}\right] x^{2}\right) \\
= & 1-\frac{l+k}{12} x+\left[\frac{2 k(l-k)}{12^{2}}+\left(\begin{array}{c}
l-k \\
2
\end{array}\right) \frac{1}{12^{2}}+\left(\begin{array}{l}
k \\
2
\end{array}\right) \frac{2}{12^{2}}+\frac{l+k}{90}\right] x^{2}
\end{aligned}
$$

and so, by integrality of the second coefficient:

$$
\begin{aligned}
& 20 k(l-k)+5(l-k)(l-k-1)+10 k(k-1)+16(l+k) \\
& \quad \equiv O\left(2^{5} \cdot 2^{2} \cdot 5\right) .
\end{aligned}
$$

Using $l-k \equiv O\left(2^{3} \cdot 3\right)$ and $B_{1}=2^{3} \cdot 3, B_{2}=2^{5} \cdot 3^{2} \cdot 5$ one easily obtains

$$
l+k \equiv O\left(2^{5}\right) ; \quad(l+k) \equiv O\left(3^{2}\right) ; \quad(l+k) \equiv O(5)
$$

5. Construction of the homotopy equivalences. To construct a stable homotopy equivalence between $\mathbf{Q} P_{k}^{n+k}$ and $\mathbf{Q} P_{l}^{n+l}$, when the arithmetical conditions permit it, we remove some of the top cells and study the attaching maps. More precisely, consider the cofibration

$$
\mathbf{Q} P_{k}^{k+t-1} \rightarrow \mathbf{Q} P_{k}^{n+k} \rightarrow \mathbf{Q} P_{t+k}^{n+k}
$$

Whenever $2(t+k)>n+k$, this cofibration desuspends to give

$$
\Sigma^{-1} \mathbf{Q} P_{t+k}^{n+k} \rightarrow \mathbf{Q} P_{k}^{k+t-1} \rightarrow \mathbf{Q} P_{k}^{n+k} \text {. }
$$

Recall, that given two cofibrations and a commutative diagram

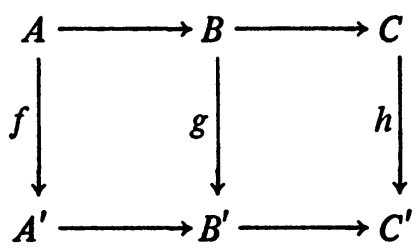

with $f$ and $g$ homotopy equivalences, there exists a homotopy equivalence $h: C \rightarrow C^{\prime}$.

With this in mind, we shall examine the cofibrations which produce the spaces $\mathbf{Q} P_{k}^{n+k}$.

First of all we shall introduce standard equivalences

$$
\mathbf{Q} P_{r+B_{n}}^{n+r+B_{n}} \rightarrow \Sigma^{4 B_{n}} \mathbf{Q} P_{r}^{n+r}
$$


This amounts to fixing a $J$-trivialization of $B_{n} \xi$ over $\mathbf{Q} P^{n}$.

Consider now the sequence

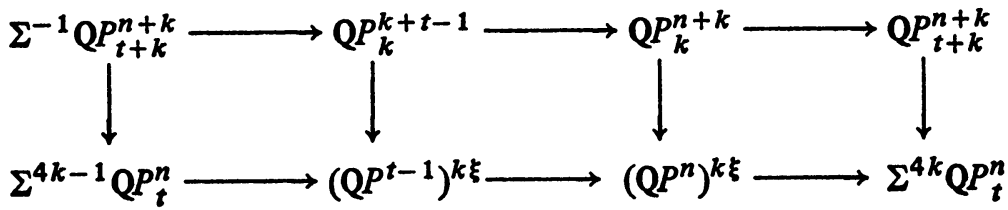

The last vertical arrow is the "standard" equivalence as long as we make sure that $k \equiv O\left(B_{n-t}\right)$.

We now restrict our attention to the space $\left(Q P^{t-1}\right)^{k \xi}$. If $k \equiv O\left(B_{t-1}\right)$ then $k \xi$ is $J$-trivial and $\left(Q P^{t-1}\right)^{k \xi}$ has the same homotopy type as $S^{4 k}$ $\vee \Sigma^{4 k} Q P^{t-1}$. It is our aim now to construct specific homotopy classes of retractions

$$
\left(\mathbf{Q} P^{t-1}\right)^{k \xi} \rightarrow S^{4 k}
$$

Let $G_{m}$ denote the space of self-homotopy equivalences of $S^{m-1}$. Then $G_{m}$ e.g. [14], is a monoid under composition with classifying space $B G_{m}$. This space classifies $(m-1)$-spherical fibrations.

The cofibration

$$
\mathrm{Q} P^{t-1} \stackrel{i}{\rightarrow} \mathrm{Q} P^{n} \stackrel{\pi}{\rightarrow} \mathrm{Q} P_{t}^{n}
$$

gives rise to an exact sequence of groups:

$$
\left[\mathbf{Q} P_{t}^{n}, B G_{4 k}\right] \stackrel{\pi^{*}}{\longrightarrow}\left[\mathbf{Q} P^{n}, B G_{4 k}\right] \stackrel{i^{*}}{\longrightarrow}\left[\mathbf{Q} P^{t-1} B G_{4 k}\right] .
$$

We shall use the symbol $[k \xi]$ for the element in $\left[Q P^{n}, B G_{4 k}\right]$ which corresponds to the spherical fibration associated with $k \xi$. If $k$ $\equiv O\left(B_{t-1}\right)$ then $i^{*}[k \xi]=0$ and so there exists an element $\alpha \in\left[Q P_{t}^{n}, B G_{4 k}\right]$ such that $\pi^{*} \alpha=[k \xi]$.

Note that $\pi$ induces a map between Thom spaces

$$
\left(\mathbf{Q} P^{n}\right)^{k \xi} \stackrel{\tilde{\pi}}{\rightarrow}\left(\mathbf{Q} P_{t}^{n}\right)^{\alpha}
$$

which we shall consider cellular. Since $\left(Q P_{t}^{n}\right)^{\alpha}$ has no cells of dimension $r, 4 k<r<4 k+4 t(4 k+4 t)$, the restriction of this map to $\left(Q P^{t-1}\right)^{k \xi}$ $\subset\left(\mathbf{Q} P^{n}\right)^{k \xi}$ yields a map

$$
\left(\mathbf{Q} P^{t-1}\right)^{k H} \stackrel{g_{\alpha}}{\longrightarrow} S^{4 k}
$$

which is clearly a retraction. The homotopy class of $g_{\alpha}$ depends only on the choice of $\alpha$. 
Let us denote by $F_{m-1} \subset G_{m}$ the submonoid of those self-equivalences of the $(m-1)$-sphere which preserve the base point. Using the suspension map we have an inclusion $G_{n} \subset F_{n}$ and this is a $(2 n-5)$-equivalence (see e.g. [10]). We have $F_{n}=\Omega^{n} S^{n}$. Suppose $X=\Sigma Y$ then, if $m>2 \operatorname{dim} X$, we have

$$
\begin{aligned}
{\left[X, B G_{m}\right] } & \cong\left[\Sigma Y, E G_{m}\right] \cong\left[Y, G_{m}\right] \cong\left[Y, F_{m}\right] \\
& \cong\left[Y, \Omega^{m} S^{m}\right] \cong\left[\Sigma^{m} Y, S^{m}\right] \cong\left[\Sigma^{m-1} X, S^{m}\right] .
\end{aligned}
$$

Let $\theta:\left[X, B G_{m}\right] \cong\left[\Sigma^{m-1} X, S^{m}\right]$. We recall the following result which appears in Wall [17, Proposition 3.7]:

Proposition 5.1. Let $\alpha$ be an $(m-1)$-spherical fibration over $\Sigma Y$, where $m>2 \operatorname{dim} \Sigma Y$. Then

$$
\Sigma^{m} Y \stackrel{\theta(\alpha)}{\longrightarrow} S^{m} \rightarrow(\Sigma Y)^{\alpha}
$$

is a cofibration.

If we assume that $2 t>n$ so that $Q P_{t}^{n}$ is a suspension then we get the cofibration

$$
\Sigma^{4 k-1} \mathbf{Q} P_{t}^{n} \stackrel{\theta(\alpha)}{\longrightarrow} S^{4 k} \rightarrow\left(\mathbf{Q} P_{t}^{n}\right)^{\alpha} .
$$

The following commutative diagram relates $\theta(\alpha)$ and $g_{\alpha}$ :

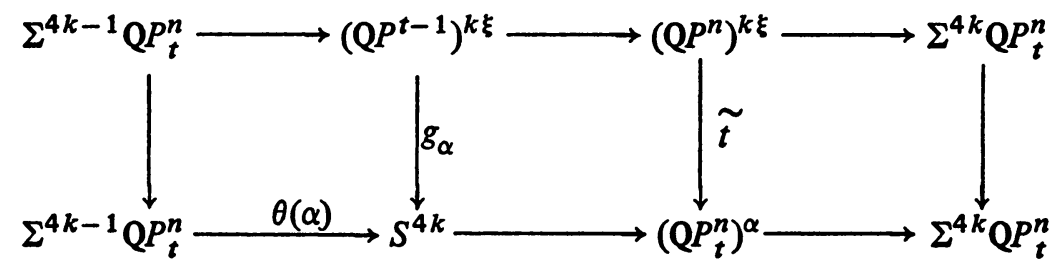

The inclusion $B G_{4 k} \subset B G_{4 k+1}$ induces isomorphisms

$$
\left[X, B G_{4 k}\right] \rightarrow\left[X, B G_{4 k+1}\right]
$$

if the dimension of $X$ is small compared to $k$. This way we can consider $\alpha \in\left[\mathbf{Q} P_{t}^{n}, B G_{4 k}\right]$ to be an element of $\left[\mathbf{Q} P_{t}^{n}, B G_{4 k+r}\right]$. All resulting maps would be suspended $r$ times.

LeMMA 5.2. Let $k \xi$ and $l \xi$ be multiples of the Hopf bundle over $\mathbf{Q} P^{n}$. If $k \xi$ as well as $l \xi$ are J-trivial over $\mathbf{Q} P^{t-1}$ and $(k+l) \xi$ is J-trivial over $\mathbf{Q} P^{n}$ then there exist $\alpha, \beta \in\left[Q P_{t}^{n}, B G_{4(k+l)}\right]$ such that $\pi^{*} \alpha=[k \xi]+4 l, \pi^{*} \beta=[l \xi]$ $+4 k$ and $\alpha+\beta=0$.

Proof. The cofibration 


$$
\mathbf{Q} P^{t-1} \stackrel{i}{\rightarrow} \mathbf{Q} P^{n} \stackrel{\pi}{\rightarrow} \mathbf{Q} P_{t}^{n}
$$

yields an exact sequence:

$$
\left[\Sigma \mathbf{Q} P^{t-1}, B G_{r}\right] \stackrel{\delta}{\rightarrow}\left[\mathbf{Q} P_{t}^{n}, B G_{r}\right] \stackrel{\pi^{*}}{\longrightarrow}\left[\mathbf{Q} P^{n}, B G_{r}\right] \stackrel{i^{*}}{\longrightarrow}\left[\mathbf{Q} P^{t-1}, B G_{r}\right]
$$

where $r=4(k+l)$.

Since $k \xi$ and $l \xi$ are $J$-trivial over $\mathbf{Q} P^{t-1}$ there exist elements $\alpha^{\prime}, \beta^{\prime}$ $\in\left[Q P_{t}^{n}, B G_{4(k+l)}\right]$ with

$$
\pi^{*} \alpha^{\prime}=[k \xi]+4 l \text { and } \pi^{*} \beta^{\prime}=[l \xi]+4 k .
$$

Since $\pi^{*}\left(\alpha^{\prime}+\beta^{\prime}\right)=0$ there exists an element $\gamma \in\left[\sum Q P^{t-1}, B G_{4(k+l)}\right]$ such that $\delta \gamma=\alpha^{\prime}+\beta^{\prime}$. Let $\alpha=\alpha^{\prime}$ and $\beta=\beta^{\prime}-\delta \gamma$.

We now are ready to construct the desired homotopy equivalences.

Proposition 5.3. If $(k-l) \xi$ is J-trivial over $\mathbf{Q} P^{n-1}$ and $(k+l) \xi$ is J-trivial over $\mathbf{Q} P^{n}$ with $n>2$ then $\mathbf{Q} P_{k}^{n+k}$ and $\mathbf{Q} P_{l}^{n+l}$ are of the same stable homotopy type.

Proof. From $k-l \equiv O\left(B_{n-1}\right)$ and $k+l \equiv O\left(B_{n}\right)$ we obtain $2 k \equiv 2 l$ $\equiv O\left(B_{n-1}\right)$ and $k \equiv l \equiv O\left(B_{[n / 2]}\right)$ for $n-1>1$. Choosing $t-1=[n / 2]$ all arguments in (5.2) go through. Let $\alpha, \beta \in\left[Q P_{t}^{n}, B G_{4(k+l)}\right]$ be the pullbacks of $[k \xi]$ and $[l \xi]$ respectively and let $\alpha+\beta=0$.

Note that $\mathbf{Q} P_{k+1}^{t-1+k}=\mathbf{Q} P_{k}^{t-1+k} / S^{4 k}$ is canonically equivalent with $\Sigma^{4 k} \mathbf{Q} P^{t-1}$. Using the collapsing map $\mathbf{Q} P_{k}^{t-1+k} \stackrel{p_{k}}{\longrightarrow} \mathbf{Q} P_{k+1}^{t-1+k}$ together with $g_{\alpha}$ we get a homotopy equivalence

$$
\left(\mathbf{Q} P^{t-1}\right)^{k H} \stackrel{g_{\alpha} \bigvee p_{k}}{\longrightarrow} S^{4 k} \vee \Sigma^{4 k} \mathbf{Q} P^{t-1} .
$$

Now consider the diagram of cofibrations and maps between them:

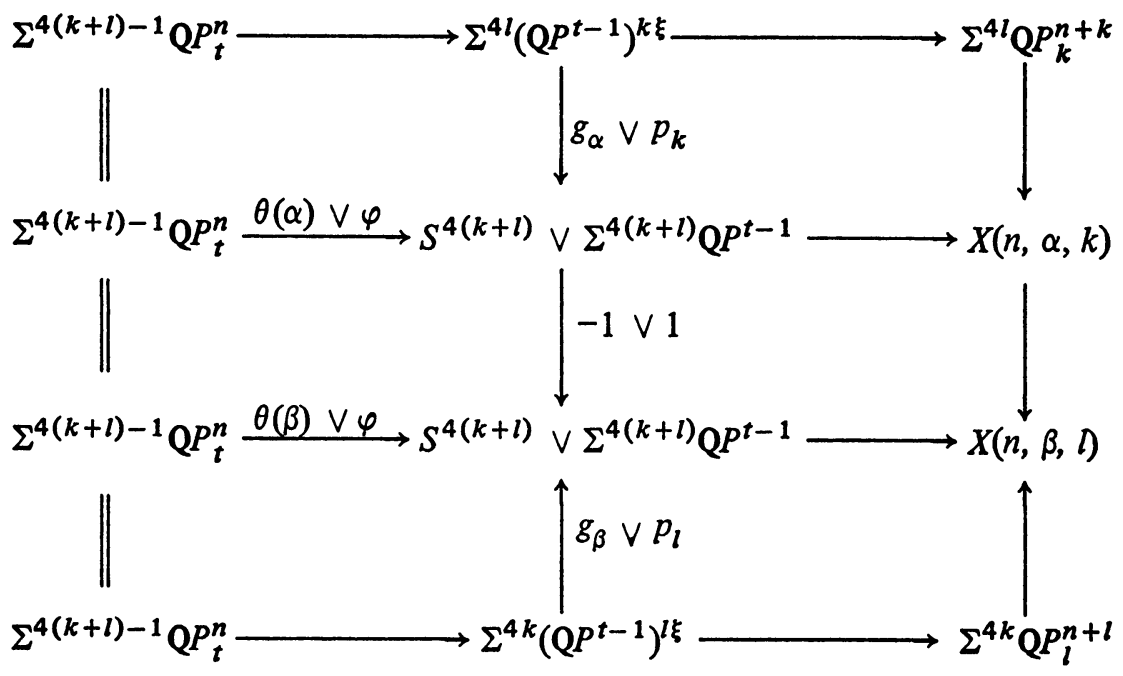


The map $\varphi$ is obtained using the canonical equivalences $\left(Q P^{t-1}\right)^{B_{t-1} \xi}$ $\sim \Sigma^{4 B_{t-1}}\left(\mathbf{Q} P^{t-1} \vee S^{0}\right)$. Since $\alpha+\beta=0$, and so $-\theta(\alpha)=\theta(\beta)$, all rectangles are commutative and all spaces on the right are of the same homotopy type.

With this proposition we complete the proof of Theorem 1.2 for $n>2$. The same argument yields the homotopy equivalences needed for Theorem 1.1.

Let $\mathcal{E}^{s}(X)$ denote the group of homotopy classes of stable self equivalences of the space $X$. There is a natural representation

$$
\mathcal{E}^{s}(X) \stackrel{e}{\longrightarrow} \text { Aut }\left(H^{*}(X ; Z)\right)
$$

where $H^{*}(X ; Z)$ is considered to be an abelian group only.

For stunted complex projective spaces we have

THEOREM 5.4. The image of $e$ for $X=\mathrm{C} P_{k}^{n+k}$ for $n>4$ is generated by

(i) $-I$ and $(-1,1,1, \ldots, 1)$ if $2 k \equiv O\left(A_{n}\right)$,

(ii) $-I$ and $(1,-1,1, \ldots, 1)$ if $k+1 \equiv O\left(1_{n-1}\right)$,

(iii) $-I$ and $(1, \ldots, 1,-1,1)$ if $k+n+2 \equiv O\left(A_{n-1}\right)$,

(iv) $-I$ and $(1, \ldots, 1,1,-1)$ if $2(k+n+1) \equiv O\left(A_{n}\right)$, and by $-I$ alone otherwise.

For stunted quaternionic projective spaces we have, similarly,

THEOREM 5.5. The image of $e$ for $X=\mathbf{Q} P_{k}^{n+k}$ is generated by $-I$ and $(-1,1,1, \ldots, 1)$ if $2 k \equiv O\left(B_{n}\right)$ and by $-I$ alone otherwise.

These theorems follow directly from the constructions of homotopy equivalences and the necessary conditions obtained via $K$-theory. Only (ii) or the dual statement (iii) of Theorem 1.1 needs a separate proof. We shall prove (ii). To this end we need only construct a self-equivalence of the form $(1,-1$, $1, \ldots, 1)$. Consider the cofribration

$$
\Sigma^{-1} \mathrm{C} P_{k+1}^{n+k} \rightarrow S^{2 k} \rightarrow \mathrm{C} P_{k}^{n+k} \text {. }
$$

Since $k+1 \equiv O\left(A_{n-1}\right)$ we have $\mathbf{C} P_{k+1}^{n+k} \sim S^{2 k+2} \vee \mathbf{C} P_{k+2}^{n+k}$ and the diagram

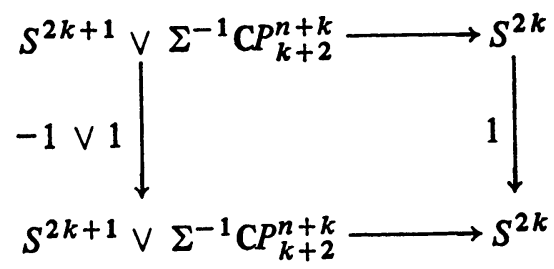

is homotopy commutative since $2 \pi_{2 k+1}\left(S^{2 k}\right)=0$. This yields the desired selfequivalence.

REMARK. This, in principle, solves the classification problem of Thom spaces over stunted complex and quaternionic projective spaces using the results of [8]. 


\section{REFERENCES}

1. J. F. Adams, On the groups $J(X)$. I, Topology 2 (1963), 181-195. MR 28 \#2553.

2. - On the groups $J(X)$. II, Topology 3 (1965), 137-172. MR 33 \#6626.

3. J. F. Adams and G. Walker, On complex Stiefel manifolds, Proc. Cambridge Philos. Soc. 61 (1965), 81-103. MR 30 \#1516.

4. M. Atiyah and J. A. Todd, On complex Stiefel manifolds, Proc. Cambridge Philos. Soc. 56 (1960), 342-353. MR 24 \# A2392.

5. R. Bott, A note on the KO-theory of sphere-bundles, Bull. Amer. Math. Soc. 68 (1962), 395-400. MR 27 \#2988.

6. S. Feder and S. Gitler, Stable homotopy types of stunted complex projective spaces, Proc. Cambridge Philos. Soc. 73 (1973), 431-438. MR 47 \# 4252.

7. , Mappings of quaternionic projective spaces, Bol. Soc. Mat. Mexicana (2) 18 (1973), 33-37. MR 49 \# 1513.

8. - Stable homotopy types of Thom complexes, Quart. J. Math. Oxford Ser. 25 (1974), 143-149. MR 50 \# 14746.

9. - Stunted projective spaces and the J-order of the Hopf bundle, Bull. Amer. Math. Soc. 80 (1974), 748-749.

10. S. Gitler and J. D. Stasheff, The first exotic class of BF, Topology 4 (1965), 227-266. MR 31 \#5215.

11. R. P. Held and D. Sjerve, On the stable homotopy type of Thom complexes, Canad. J. Math. 25 (1973), 1285-1294. MR 49 \#3911.

12. K. Y. Lam, Fiber homotopic trivial bundles over complex projective spaces, Proc. Amer. Math. Soc. 33 (1972), 211-212. MR 45 \#2731.

13. D. Quillen, The Adams conjecture, Topology 10 (1970), 67-80. MR 43 \#5525.

14. B. Sanderson, Immersions and embeddings of projective spaces, Proc. London Math. Soc. (3) 14 (1964), 137-153. MR 29 \#2814.

15. F. Sigrist and U. Suter, Cross-sections of symplectic Stiefel manifolds, Trans. Amer. Math. Soc. 184 (1973), 247-259. MR 48 \#5071.

16. J. D. Stasheff, $A$ classification theorem for fiber spaces, Topology 2 (1963), 239-246. MR 27 \#4235.

17. C. T. C. Wall, Poincaré complexes. I, Ann. of Math. (2) 86 (1967), 213-245. MR 36 \#880.

Departamento de Matematicas, Centro de Investigacion del Instituto Politecnico Nacional, Mexico, D. F., Mexico 